\title{
Case Blog
}

\section{Title: Spontaneous Osteonecrosis as a Cause of Knee Pain in an Older Active Woman}

\section{Lance M. Mabry ${ }^{1}$, Michael D. Ross ${ }^{2 *}$, Kristi A. Greene ${ }^{3}$ and Michael A. Tall ${ }^{4}$}

${ }^{1}$ Medical Group, Joint Base Andrews, USA

${ }^{2}$ Daemen College, Amherst NY, USA

${ }^{3}$ Agile Physical Therapy, Palo Alto, CA, USA

${ }^{4}$ San Antonio Uniformed Health Educational Consortium, San Antonio, TX, USA
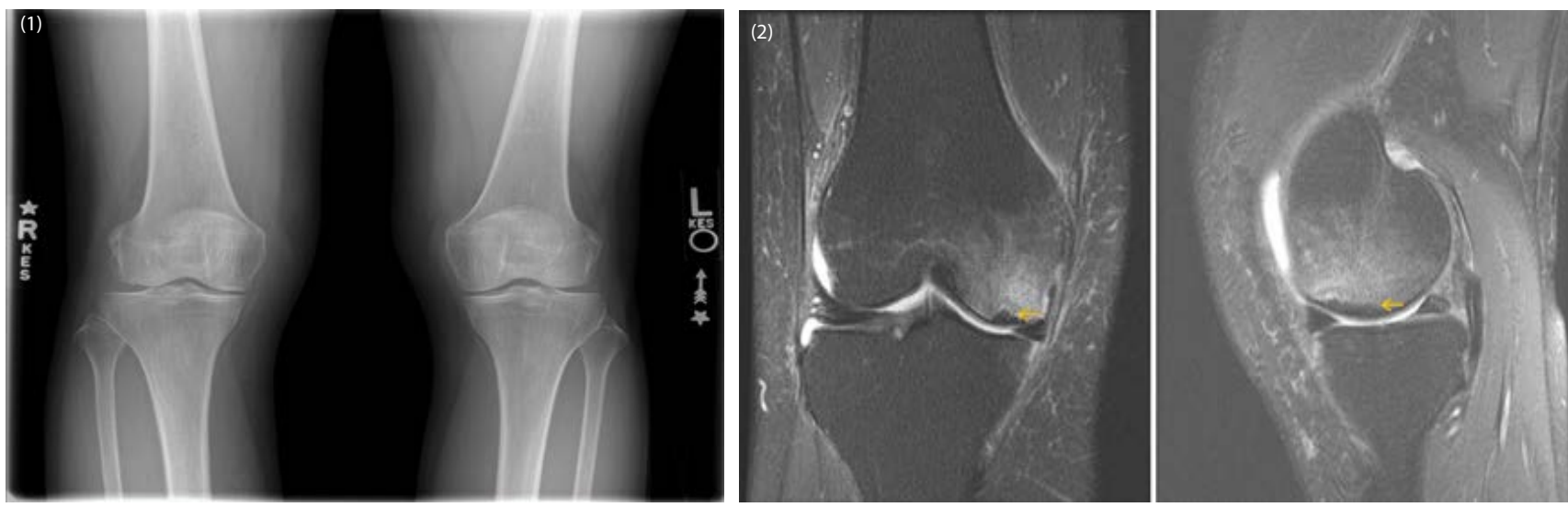

Figure 1: Bilateral knee weight bearing anterior to posterior view radiograph revealing minimal medial joint space narrowing on the right.

Figure 2: T2 weighted (fat saturated) magnetic resonance imaging (left: coronal view; right: sagittal view) demonstrated an area of low signal intensity in the subchondral portion of the medial femoral condyle with a curvilinear serpiginous low signal intensity line in the subchondral bone likely from subchondral fracture and ischemia (arrows). There is considerable surrounding bone marrow edema.

The patient was a 63 year old woman with a chief complaint of worsening right knee pain, which began 6 weeks prior while walking down a steep incline. Prior to symptom onset, the patient ran $6.5 \mathrm{~km}$ daily. The patient's symptoms initially presented as tightness in the posterior aspect of the knee, but changed to a constant varying ache along the medial aspect of the knee with occasional sharp pain during ambulation. The patient also reported night pain and as well as morning stiffness. Radiographic findings were significant for mild degenerative changes with minimal medial joint space narrowing for the right knee (Figure 1). The patient was treated with a corticosteroid injection, which only provided temporary minimal relief. Magnetic resonance imaging was subsequently ordered and revealed findings consistent with spontaneous osteonecrosis of the medial femoral condyle (Figure 2), which is a distinct form of osteonecrosis and usually demonstrates a subchondral fracture identifiable on magnetic resonance imaging. This can affect males or females but it more commonly affects older women and is typically characterized by the sudden onset of knee pain [1]. In general, the lesion is more commonly located in a femoral condyle and it is not usually associated with systemic disorders or previous corticosteroid therapy [1]. The patient attempted a course of conservative treatment including protected weightbearing with hyaluronic acid, and physical therapy. However, the patient did not respond to conservative management and is awaiting unicompartmental knee arthroplasty.

Note: The opinions or assertions contained herein are the private views of the authors and are not to be construed as official or reflecting the views of the US Air Force or Department of Defense.

\section{References}

1. Zywiel MG, McGrath MS, Seyler TM, Marker DR, Bonutti PM, et al. (2009) Osteonecrosis of the knee: a review of three disorders. Orthop Clin North Am 40: 193-211. 